\title{
Design and Evaluation of Famotidine Mucoadhesive Nanoparticles for Aspirin Induced Ulcer Treatment
}

\author{
Dhaval J Patel $^{1 *}$ and Jayvadan K Patel $^{2}$ \\ ${ }^{I}$ Department of Pharmaceutics; Saraswati Institute of Pharmaceutical Sciences; Gandhinagar; 382355, Gujarat, \\ India. ${ }^{2}$ Department of Pharmaceutics; Nootan Pharmacy College; Visnagar, 384315, Gujarat, India.
}

\begin{abstract}
The present study was performed to design and evaluate the famotidine loaded mucoadhesive nanosuspension for aspirin induced ulcer. A 3-factor, 3-level Box-Behnken design was applied to study the effects of amount of the beads $\left(X_{1}\right)$, PVPK-30( $\left.X_{2}\right)$ and Tween-80 $\left(X_{3}\right)$ on the particle size $\left(Y_{1}\right)$, and cumulative percentage drug released after $1 \mathrm{~h}\left(\mathrm{Y}_{2}\right)$. The optimization was performed using the desirability function and contour plots. The scanning electron microscopy (SEM) showed the nanoparticles as spherical in shape. The differential scanning calorimetry (DSC) analysis indicated that there was substantial crystallinity change in the nanoparticle compared with the pure drug. Ex-vivo mucoadhesion study showed that famotidine mucoadhesive nanoparticles possessed higher mucoadhesion than the famotidine nanoparticles. The in vivo studies on aspirin-induced rats indicated the lowering in ulcer index for famotidine mucoadhesive nanoparticles was 0.46+0.011, which was significantly better than the effect of traditional famotidine suspension (0.66+0.035). Famotidine mucoadhesive nanosuspension could be prepared using the media milling technique and allowing significant reduction in ulcer index compared to famotidine suspension.
\end{abstract}

Key words: Famotidine, Nanosuspension, Box-Behnken design, Mucoadhesion, peptic ulcer

\section{INTRODUCTION}

Among various noxious substances such as acid, pepsin, H. pylori, the use of non steroidal antiinflammatory drugs (NSAIDs) plays a major role in the pathogenesis of peptic ulcer disease. The use of NSAIDs is the second most common cause of ulcers and the rate of NSAID-caused ulcers is increasing (Ibrahim et al. 2007; Hawkey (1990); Derle et al. 2006). Famotidine is $\mathrm{H}_{2}$-receptor antagonist widely prescribed in the gastric ulcers, duodenal ulcers, Zollinger-Ellison syndrome and gastro-esophageal reflux disease. Famotidine is reported to be 7.5 and 20 times more potent than ranitidine and cimetidine, respectively. Famotidine is classified as BCS class IV substance, since, drug of this class exhibits poor and variable bioavailability. The overall bioavailability is governed by several factors such as the rate of dissolution, intestinal permeability, gastric emptying and so on (Hassan et al.1990). A number of drugs remain poorly available when administered by the oral route. Among other reasons, this fact can be related either to, a low mucosal permeability for the drug or low solubility for the drug in the mucosal fluids. In both the cases, the drug absorption is poor, and an important fraction of the given dose is eliminated from the alimentary canal before being absorbed (Arangoa et al. 2001). The variability of absorption could also be reduced by their tendency

*Author for correspondence: dhaval6668@gmail.com 
to adhere to the gastrointestinal wall enabling rapid replenishment of the absorbed drug. To circumvent these problems, the drug nanoparticles system treated with mucoadhesive polymers has been proposed. These small particles have ability to penetrate in the mucus layer and bind to underlying epithelium and adhere directly to the mucus network. Thus, it may be expected that the particle uptake into the disrupted barrier in gastric ulcerations could allow the accumulation of the particulate carrier system in the desired area. Hence, subsequent increase in local drug concentration, prolongs the residence time of the drug in the gut and, therefore, increases the time when absorption can occur (Hasani et al. 2009).

Hence, the aim of present work was done to formulate and evaluate famotidine mucoadhesive nanosuspension for aspirin induce peptic ulcer treatment to overcome the problem of less contact time in ulceration. Famotidine nanosuspension was prepared using the media milling technique. Box-Behnken design was used to optimize the nanosuspension containing famotidine. The relationship between the dependent and independent variables was constructed using the contour plots. Then, the experimental design was combined with the desirability functions to predict the desired quality. The optimized famotidine nanoparticles were incorporated in physical admixture of mucoadhesive polymers (Hydroxyl propyl methyl cellulose/Sodium carboxymethylcellulose) dispersion to prolong their therapeutic effect. These combined systems were evaluated for mucoadhesion, rheological behavior, in vitro drug release and in vivo study.

\section{MATERIALS AND METHODS}

Famotidine was obtained as a gift sample from Cadila Pharmaceutical, Gujarat, India. Polyvinylpyrrolidone (PVPK-30) was purchased from Sisco Research Laboratory, India. Tween-80 and Sodium carboxymethylcellulose (Na-CMC) were gifted by the S.D Fine Chemical Ltd, Mumbai, India. Hydroxyl propyl methyl celluloseK4M (HPMC-K4M) was obtained as a gift sample from the Colorcon Asia Ltd, Goa, India. Double distilled water was prepared in the laboratory. All the chemicals used in the study were of analytical grade and used without further purification.

\section{Box-Behnken experimental design}

A Box-Behnken statistical design with three factors, three levels, and 15 runs was selected for the optimization study (Solanki et al. 2007). The experimental design consisted of a set of points lying at the midpoint of each edge and the replicated center point of the multidimensional cube. The independent and dependent variables are listed in Table 1 . The polynomial equation generated by this experimental design (Microsoft excel, 2007) was as follows

$Y i=b_{0}+b_{1} X_{1}+b_{2} X_{2}+b_{3} X_{3}+b_{12} X_{1} X_{2}+b_{13} X_{1} X_{3}+b_{23} X_{2}$ $\mathbf{X}_{3}+\mathbf{b}_{11} \mathbf{X}_{1}{ }^{2}+\mathbf{b}_{22} \mathbf{X}_{2}^{2}+\mathbf{b}_{33} \mathbf{X}_{3}^{2}$

Here, Yi was the dependent variable, $b_{0}$ was the intercept, $b_{1}$ to $b_{33}$ were the regression coefficients and $X_{1}, X_{2}$ and $X_{3}$ were the independent variables that were selected from the preliminary experiments. After the fitting of the mathematical model, the desirability function was used for the optimization. The application of the desirability function combined all the responses into one variable and left the possibility to predict the optimum levels for the independent variables (Holm et al. 2006).

\section{Preparation of nanosuspension}

Nanosuspensions were prepared by the media milling method (Pandya et al. 2010). Zirconium oxide beads $(\varnothing 0.5 \mathrm{~mm})$ were used as the milling media to prepare the aqueous nanosuspensions of famotidine at fixed concentration in $20 \mathrm{ml}$ vials. All the batches were prepared according to the experimental design as shown in Table.1. The independent variables and their amounts were selected from the preliminary screening study (Patel et al. 2010). Also, in $20 \mathrm{ml}$ vials, PVPK-30 was used as the polymeric stabilizer along with Tween-80 as non-ionic surfactant. The comminuting process was performed on a highspeed shaker (Remi, India) at $250 \mathrm{rpm}$ at room temperature for $24 \mathrm{~h}$. After milling, nanosuspensions were separated from the zirconium beads by decanting the suspension followed by washing of the beads with double distilled water. Milling was carried out under ambient conditions throughout the experiment. 
Table 1 - Variables and their levels in Box-Behnken design.

\begin{tabular}{lccc}
\multicolumn{1}{c}{ Independent Variables } & \multicolumn{2}{c}{ Levels } \\
\cline { 2 - 4 } & Low & Medium & High \\
\hline $\mathbf{X}_{\mathbf{1}}:$ Amount of zirconium oxide beads $(\mathbf{g m})$ & 6 & 7 & 8 \\
$\mathbf{X}_{\mathbf{2}}$ : Amount of PVPK-30 $(\mathbf{m g})$ & 40 & 60 & 80 \\
$\mathbf{X}_{\mathbf{3}}$ : Amount of tween-80 $(\mathbf{m l})$ & 0.06 & 0.08 & 0.1 \\
Transform values & Dependent variables & & 0 \\
\end{tabular}

$\mathrm{Y}_{1}:$ Particle size (nm)

$Y_{2}: Q_{1}(\%$ drug release after $1 \mathrm{~h})$

\section{Characterization of nanoparticles \\ Particle size and zeta potential analysis}

Particle size analysis of the nano-suspension formulations was performed by the photon correlation spectroscopy using a Zetasizer 3000 (Malvern Instruments, UK). The formulations were added drop-wise to the sample dispersion unit containing water (saturated with famotidine) as a dispersant. A refractive index value of 1.5 was used for particle size analysis. Also, zeta potential for the optimized nano-suspension in distilled water was determined using the same equipment. All the data presented are the mean values of the results on three independent samples produced under the identical conditions.

\section{SEM study}

The surface morphologies of the micronized famotidine and optimized famotidine nanoparticles were visualized by the SEM. Particle morphology was investigated using a Hitachi (S-4700, Japan) with an acceleration voltage of $30 \mathrm{kV}$ followed by coating with Pt-Pd for 2 min.

\section{Preparation of famotidine loaded mucoadhesive nanosuspension}

Table 2 shows the composition of all the mucoadhesive nano-suspensions. The HPMC$\mathrm{K} 4 \mathrm{M}$ and $\mathrm{Na}-\mathrm{CMC}$ were dispersed gradually in separate container using double distilled water with slow magnetic stirring (Remi, India). Then, mucoadhesive dispersions were prepared by mixing the weighed quantities of Na-CMC $(0.2$, 0.4 and $0.6 \% \mathrm{w} / \mathrm{v}$ ) solutions with the HPMC-K4M $(0.6,0.4$ and $0.2 \%)$ solutions in $50 \mathrm{ml}$ glass beaker left to equilibrate for $24 \mathrm{~h}$. The famotidine nanoparticles were then added to the HPMC-K4M and $\mathrm{Na}-\mathrm{CMC}$ solution under constant stirring to obtain the uniform mixture and same for their polymer mixtures.

Table 2 - Formulation of famotidine mucoadhesive nanosuspensions.

\begin{tabular}{lcccccccccc}
\hline \multicolumn{1}{c}{ Ingredients } & $\mathbf{B}_{\mathbf{1}}$ & $\mathbf{B}_{\mathbf{2}}$ & $\mathbf{B}_{\mathbf{3}}$ & $\mathbf{B}_{\mathbf{4}}$ & $\mathbf{B}_{\mathbf{5}}$ & $\mathbf{B}_{\mathbf{6}}$ & $\mathbf{B}_{\mathbf{7}}$ & $\mathbf{B}_{\mathbf{8}}$ & $\mathbf{B}_{\mathbf{9}}$ & $\mathbf{B}_{\mathbf{1 0}}$ \\
\hline $\begin{array}{l}\text { Optimized nanosuspension equivalent to } \\
\text { 40 mg of famotidine(ml) }\end{array}$ & 10 & 10 & 10 & 10 & 10 & 10 & 10 & 10 & 10 & 10 \\
HPMCK4M (\% w/v) & & & & & & & & & & \\
Na-CMC (\% w/v) & 0.6 & 0.4 & 0.2 & 0.6 & 0.8 & 1 & & & & \\
Volume of aqueous solvent & 0.2 & 0.4 & 0.6 & & & & 0.6 & 0.8 & 1 & \\
Stirring Speed( Rpm) & 10 & 10 & 10 & 10 & 10 & 10 & 10 & 10 & 10 & \\
& 600 & 600 & 600 & 600 & 600 & 600 & 600 & 600 & 600 & 600 \\
\hline
\end{tabular}

\section{Rheological measurement}

The viscosity of the mucoadhesive nanosuspensions was determined by the Brookfield viscometer (LV/M/00-151-E0808, MA, USA). Mucoadhesive nanosuspensions were filled in clean and dry test tube (Capacity $25 \mathrm{ml}$ ). The viscosity of the mucoadhesive nanosuspensions were determined at various rpm $6,12,30$ and 60 using spindle no 3 and was calculated using the dial reading with its respective factor in repeated manner.

\section{Mucoadhesion measurement}

The shear stress measurement method, based on the measurement of shear stress required to break the adhesive bond between glass slides and the test formulation was used. The test formulation was sandwiched between the two glass slides fixed on flexible supports in the assemblies for a sufficient period of time. Water from a burette was allowed to fall in a beaker at a constant rate of $10 \mathrm{ml} / \mathrm{sec}$. Increasing weight of water added gradually detached the two glass slides. Mucoadhesive force, 
the detachment stress $(\mathrm{g})$, was determined from the minimal weights of water that detached the glass slides. The experiments were carried out in triplicates (Verma et al. 2009; Sheikh et al. 2009).

\section{Thermal analysis}

The optimized mucoadhesive nanodispersion was centrifuge at $10,000 \mathrm{rpm}$ for $10 \mathrm{~min}$ and the particle pellet was oven-dried at $50^{\circ} \mathrm{C}$ for $24 \mathrm{~h}$. Afterwards, it was used for the DSC analysis. The DSC scans of the famotidine and mucoadhesive nanoparticulate dispersion were recorded using the DSC-Shimadzu 60 (Shimadzu Co., Kyoto, Japan) with TDA trend line software. All the samples were weighed $(8-10 \mathrm{mg})$ and heated at a scanning rate of $20^{\circ} \mathrm{C} / \mathrm{min}$ under dry air flow $(100 \mathrm{ml} / \mathrm{min})$ between 50 and $300^{\circ} \mathrm{C}$ using the aluminum pans and lids for all the samples.

\section{Drug release study}

A double- jacketed beaker was used as a dialysis system to study the dissolution behavior of mucoadhesive nano-suspensions. The dissolution medium used was freshly prepared using $0.1 \mathrm{~N}$ HCL ( $\mathrm{pH}$ 1.2). Dialysis membrane, previously soaked overnight in the dissolution medium was tied to one end of a specifically designed glass cylinder (open at both the ends) (Pandya et al. 2010; Lai et al. 2009). Five milliliter of the formulation was accurately placed into this assembly. The cylinder was attached to a stand and suspended in $300 \mathrm{ml}$ of dissolution medium maintained at $37 \pm 1^{\circ} \mathrm{C}$ so that the membrane just touched the receptor medium surface. The contents of the beaker were agitated on a magnetic stirrer. Five milliliter of the sample was withdrawn periodically and replaced with an equal volume of fresh $0.1 \mathrm{~N} \mathrm{HCl}(\mathrm{pH} 1.2)$ up to $8 \mathrm{~h}$. The samples were diluted suitably and filtered through a filter paper $(0.22 \mu \mathrm{m}$, Whatman Inc., USA). The sample was then subject to the UV analysis against the blank $(0.1 \mathrm{NHCl}$ solution). Percent cumulative release of famotidine was calculated based on the standard UV calibration curve at $267 \mathrm{~nm}$ (Systronic 2203, Japan).

\section{Ex-vivo retention of mucoadhesive nano- suspension}

Ex-vivo retention of mucoadhesive nanosuspension was adopted from the previously reported method (Rajinikanth et al. 2008). The approval of the Institutional Animal Ethics
Committee was obtained before the starting the study. The stomach of the overnight fasted rats was removed and cut into pieces $(2 \mathrm{~cm}$ long and 1 $\mathrm{cm}$ wide) and were rinsed with $2.0 \mathrm{ml}$ of physiological saline and $5.0 \mathrm{ml}$ of optimized mucoadhesive nanoparticulate dispersion. The nanoparticulate dispersion of drug were placed uniformly on the surface of the stomach mucosa and allowed to react with mucin for $15-20 \mathrm{~min}$. The mucosal lumen was rinsed with $\mathrm{HCl}$-physiological saline at angle of $45^{\circ}$. The amount of formulation, which remained coated onto the tissues after rinsing in $\mathrm{HCl}$-physiological saline was determined.

\section{In vivo study}

All the animal experiments were carried out in accordance with the recommendations of the Institutional Animal Ethics Committee of Shree Krishna Institute of Pharmacy Shankhalpur, Gujarat, India. Albino rats of both sexes (age:- 1012 weeks and weighing 200-250 g) were used in all the experiments. Each group of the treated animals contained at least three rats and was housed in standard cages. Disease control (distilled water-10 ml $/ \mathrm{kg}$ ), famotidine marketed suspension $(40 \mathrm{mg} / 10 \mathrm{ml} / \mathrm{kg}$ ), and famotidine mucoadhesive nano-suspension (B3) (40 $\mathrm{mg} / 10 \mathrm{ml} / \mathrm{kg})$ were arranged in groups 1,2 and 3 , respectively. Distilled water, famotidine and formulation $\mathrm{B}_{3}$ were given orally and $30 \mathrm{~min}$ later aspirin (200mg/kg-per oral) was administrated to all the groups. Eight hours later, the animals were killed by decapitation. The stomachs were removed, opened along the great curvature and washed with the tap water to remove the gastric contents, then examined under a dissecting microscope with square-grid eyepiece to assess the formation of ulcers. For each stomach, ulcerated and total areas were measured as $\mathrm{mm}^{2}$. The ulcer indexes for each stomach were calculated using the following formula:

Ulcer Index $=$ [Ulcerated area $/$ Total stomach area] $* 100$

\section{Stability study}

The accelerated stability study was carried out according to the International Conference on Harmonization (ICH) guidelines (Shaikh et al. 2009). Sealed vials $(10 \mathrm{ml})$ of freshly prepared $B_{3}$ formulation and famotidine nano-suspension were placed in stability chamber maintained at 
$25^{\circ} \mathrm{C} \pm 2{ }^{\circ} \mathrm{C} / 60 \%$ relative humidity (RH). The nanoparticles subjected to comparative stability tests were analyzed over three month's period for physical appearance, particle size and sedimentation of nanoparticles at frequency of 1 month sampling. In vitro release study of formulation $\mathrm{B}_{3}$ was also carried out after every one month.

\section{RESULT AND DISCUSSION}

\section{Box-Behnken design and desirability function}

From the preliminary experimentation, it was observed that the amount of the beads, amount of PVPK-30 and amount of tween-80 contributed much towards the change in $\mathrm{Y}_{1}$ and $\mathrm{Y}_{2}$ in nanosuspension preparation using the media milling method. Therefore, Box-Behnken design was applied in this study to optimize the famotidine nano-suspension with the constraints on the particle size and $\mathrm{Q}_{1}$. The transformed values of all the batches along with their results are shown in Table 3 (Solanki et al. 2007). The polynomial equations were used to draw the conclusions after considering the magnitude of coefficient and the mathematical sign it carried (i.e., positive or negative). The mathematical relationship in the form of factor's coefficients, its corresponding Pvalues for the measured responses and correlation coefficient are listed in Table 4. Coefficients with P-value less than 0.05 had a significant effect on the prediction efficacy of the model for the measured response. The high values of correlation coefficient for the dependent variables indicated a good fit. The particle size (dependent variable, $\mathrm{Y}_{1}$ ) obtained at various levels of the three independent variables $\left(\mathrm{X}_{1}, \mathrm{X}_{2}\right.$, and $\left.\mathrm{X}_{3}\right)$ was subjected to multiple regression to yield a second-order polynomial equation (full model).

Table 3 - Formulation and dissolution characteristics of Box-Behnken design batches.

\begin{tabular}{|c|c|c|c|c|c|c|c|c|}
\hline \multirow[t]{2}{*}{ Run } & \multicolumn{3}{|c|}{ Coded value } & \multicolumn{3}{|c|}{ Actual value } & \multicolumn{2}{|c|}{ Dependent Variables } \\
\hline & $\mathbf{X}_{1}$ & $\mathbf{X}_{2}$ & $\mathbf{X}_{3}$ & $\mathbf{X}_{1}$ & $\mathbf{X}_{2}$ & $\mathbf{X}_{3}$ & $\mathrm{Y}_{1}(\mathrm{~nm} \pm$ S.D $)$ & $\mathrm{Y}_{2}(\% \pm \mathrm{S} . \mathrm{D})$ \\
\hline $\mathbf{W}_{21}$ & -1 & -1 & 0 & 6 & 40 & 0.08 & $742.0 \pm 10.80$ & $78.59 \pm 1.57$ \\
\hline $\mathbf{W}_{22}$ & 1 & -1 & 0 & 8 & 40 & 0.08 & $730.0 \pm 6.00$ & $83.91 \pm 1.83$ \\
\hline $\mathbf{W}_{23}$ & -1 & 1 & 0 & 6 & 80 & 0.08 & $525.0 \pm 10.73$ & $81.09 \pm 2.48$ \\
\hline $\mathbf{W}_{24}$ & 1 & 1 & 0 & 8 & 80 & 0.08 & $677.0 \pm 9.00$ & $81.18 \pm 1.84$ \\
\hline $\mathbf{W}_{25}$ & -1 & 0 & -1 & 6 & 60 & 0.06 & $528.0 \pm 5.32$ & $82.59 \pm 1.99$ \\
\hline $\mathbf{W}_{26}$ & 1 & 0 & -1 & 8 & 60 & 0.06 & $614.0 \pm 7.27$ & $87.19 \pm 2.99$ \\
\hline $\mathbf{W}_{27}$ & -1 & 0 & 1 & 6 & 60 & 0.1 & $448.9 \pm 7.32$ & $85.25 \pm 3.21$ \\
\hline $\mathbf{W}_{28}$ & 1 & 0 & 1 & 8 & 60 & 0.1 & $463.7 \pm 3.99$ & $89.74 \pm 2.52$ \\
\hline $\mathbf{W}_{29}$ & 0 & -1 & -1 & 7 & 40 & 0.06 & $560.0 \pm 5.80$ & $81.67 \pm 1.36$ \\
\hline $\mathbf{W}_{30}$ & 0 & 1 & -1 & 7 & 80 & 0.06 & $394.1 \pm 10.16$ & $84.87 \pm 3.55$ \\
\hline $\mathbf{W}_{31}$ & 0 & -1 & 1 & 7 & 40 & 0.1 & $432.5 \pm 12.93$ & $88.79 \pm 3.71$ \\
\hline $\mathbf{W}_{32}$ & 0 & 1 & 1 & 7 & 80 & 0.1 & $435.7 \pm 12.40$ & $85.18 \pm 4.77$ \\
\hline $\mathbf{W}_{33}$ & 0 & 0 & 0 & 7 & 60 & 0.08 & $498.0 \pm 11.18$ & $85.24 \pm 1.28$ \\
\hline $\mathbf{W}_{34}$ & 0 & 0 & 0 & 7 & 60 & 0.08 & $542.0 \pm 10.98$ & $84.14 \pm 3.41$ \\
\hline $\mathbf{W}_{35}$ & 0 & 0 & 0 & 7 & 60 & 0.08 & $532.0 \pm 13.37$ & $83.15 \pm 1.96$ \\
\hline
\end{tabular}

$\mathrm{Y}_{1}$ is indicating particle size $(\mathrm{nm})$, whereas $\mathrm{Y}_{2}$ is Cumulative percentage release of drug after $1 \mathrm{~h} . \mathrm{X}_{1}=$ amount of amount of beads, and $\mathrm{X}_{2}=$ amount of PVPK-30, $\mathrm{X}_{3}=$ amount of Tween-80. Each batch contains $40 \mathrm{mg}$ of famotidine; total quantity of nanosuspension was made to $10 \mathrm{ml}$. S.D is indicating standard deviation of the responses measured value.

$Y_{1}=524+30.10 X_{1}-54.09 X_{2}-39.39 X_{3}+41.00 X_{1} X_{2}-$ 7.30X $X_{1} X_{3}+42.30 X_{2} X_{3}+101.29 X_{1}^{2}+43.20 X_{2}^{2}-$ 111.64X $\mathrm{X}^{2}$ [2]

The particle size values measured for the different batches showed wide variation (i.e., values ranged from a minimum of 394.1 to a maximum of 742.0). The results clearly indicated that the particle size value was strongly affected by the variables selected for the study. This was also reflected by the wide range of values for the coefficients of the terms of equation 2 . The main effects of $X_{1}, X_{2}$, and $X_{3}$ represented the average result of changing one variable at a time from its low level to its high level. The interaction terms $\left(\mathrm{X}_{1} \mathrm{X}_{2}, \mathrm{X}_{1} \mathrm{X}_{3}, \mathrm{X}_{2} \mathrm{X}_{3}, \mathrm{X}_{1}^{2}, \mathrm{X}_{2}^{2}\right.$, and $\left.\mathrm{X}_{3}^{2}\right)$ showed how the particle size changed when two variables were simultaneously changed. Concerning the particle size, the results of multiple linear regression analysis showed that both the coefficients $b_{1}$ had positive sign while $b_{2}$ and $b_{3}$ had a negative sign $\left(\mathrm{R}^{2}=0.9673\right)$. Equation 2 revealed $\mathrm{X}_{1}$ had the largest positive effect compared to $\mathrm{X}_{2}$ and $\mathrm{X}_{3}$. 
Among, the interaction terms, only $b_{13}$ was nonsignificant $(\mathrm{P}>0.05)$ (Table 4$)$. The significance level of coefficients $b_{12} b_{13}$, and $b_{22}$ was $p=0.052$, 0.322 , and 0.05 respectively, hence it was omitted from the full model to generate the reduced model. Coefficients $b_{1}, b_{2}, b_{3}, b_{23}, b_{11}$ and $b_{33}$ were significant at $p<0.05$ hence, they were retained in the reduced model. The reduced model was tested in portions to determine whether the coefficients $b_{2}$ and $b_{12}$ contributed significant information for the prediction of $Y_{1}$. The results of testing the model for ANOVA are shown in Table 5. The observed and predicted values with residuals and percent error of responses for $Y_{1}$ are shown in
Table 6. The critical value of $f=5.41(p=0.05)$ was higher than the calculated value $(\mathrm{F}=4.70)$.

It could be concluded that the interaction terms $b_{12}$ $b_{13}$, and $b_{22}$ did not contribute significantly to the prediction of $Y_{1}$, and the low coefficients for these terms in equation 2 indicated that these terms contributed the least to the prediction of $Y_{1}$. Hence, these terms were omitted from the full model to obtain a reduced second-order polynomial equation 3 by multiple regressions of $\mathrm{Y}_{1}$ and the significant terms $(p<0.05)$ of equation $2, \quad Y_{1}=524+30.10 X_{1}-54.09 X_{2^{-}}$ $39.39 X_{3}+42.30 X_{2} X_{3}+101.29 X_{1}^{2}-111.64 X_{3}^{2}$

Table 4- Summary of regression analysis for measured responses.

\begin{tabular}{lccccccccccc}
\hline Coeffici & $\mathbf{b}_{\mathbf{0}}$ & $\mathbf{b}_{\mathbf{1}}$ & $\mathbf{b}_{\mathbf{2}}$ & $\mathbf{b}_{\mathbf{3}}$ & $\mathbf{b}_{\mathbf{1 2}}$ & $\mathbf{b}_{\mathbf{1 3}}$ & $\mathbf{b}_{\mathbf{2 3}}$ & $\mathbf{b}_{\mathbf{1 1}}$ & $\mathbf{b}_{\mathbf{2 2}}$ & $\mathbf{b}_{\mathbf{3 3}}$ & $\mathbf{R}^{\mathbf{2}}$ \\
\hline $\mathbf{Y}_{\mathbf{1}}$ & 524 & 30.1 & -54.09 & -39.39 & 41 & -17.8 & 42.3 & 101.29 & 43.2 & -111.64 & 0.9673 \\
P-Value & $1.1 \mathrm{e}-06$ & 0.047 & 0.005 & 0.018 & 0.052 & 0.322 & 0.048 & 0.001 & 0.05 & 0.001 & \\
$\mathbf{Y}_{\mathbf{2}}$ & 84.17 & 1.81 & -0.08 & 1.58 & -1.31 & -0.03 & -1.71 & -0.96 & -2.02 & 2.97 & 0.9639 \\
$\mathbf{P - V a l u e}$ & $2.2 \mathrm{e}-10$ & 0.003 & 0.821 & 0.005 & 0.031 & 0.956 & 0.016 & 0.011 & 0.009 & 0.001 & \\
\hline
\end{tabular}

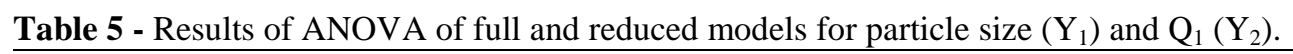

\begin{tabular}{|c|c|c|c|c|c|c|}
\hline \multicolumn{7}{|c|}{ For Particle size $\left(Y_{1}\right)$} \\
\hline Regression & df & SS & MS & $\mathbf{R}^{2}$ & p-value & $D f(3,5)$ \\
\hline FM & 9 & 156301.3 & 17366.81 & 0.9673 & 0.0033 & Fcalc $=4.70 /$ Ftab $=5.41$ \\
\hline $\mathbf{R M}$ & 6 & 141416.8 & 23569.47 & 0.8752 & 0.002946 & \\
\hline Residual & df & SS & MS & & & \\
\hline FM & 5 & 5272.871 & 1054.574 & & & \\
\hline $\mathbf{R M}$ & 8 & 20157.36 & 2519.669 & & & \\
\hline \multicolumn{7}{|c|}{ For $Q_{1}\left(Y_{2}\right)$} \\
\hline Regression & df & SS & MS & $\mathbf{R}^{2}$ & p-value & $D f(3,5)$ \\
\hline FM & 9 & 120.2303 & 13.35892 & 0.9639 & 0.004202 & Fcalc $=1.27 /$ Ftab $=5.41$ \\
\hline $\mathbf{R M}$ & 6 & 116.7762 & 19.4627 & 0.9362 & 0.000223 & \\
\hline Residual & $\mathrm{df}$ & SS & MS & & & \\
\hline FM & 5 & 4.498967 & 0.899793 & & & \\
\hline $\mathbf{R M}$ & 8 & 7.953069 & 0.994134 & & & \\
\hline
\end{tabular}

$Q_{1}$ is indicating Cumulative percentage release of drug after $1 \mathrm{~h}$.

Table 6 - Observed and predicted values with residuals of the response $\mathrm{Y}_{1}$.

\begin{tabular}{cccccc}
\hline Run & Observed $\mathbf{Y}_{\mathbf{1}}(\mathbf{n m})$ & PI \pm S.D of Y1 & Predicted $\mathbf{Y}_{\mathbf{1}}(\mathbf{n m})$ & Residuals & \%Errors \\
\hline $\mathbf{W}_{\mathbf{2 1}}$ & $742.0 \pm 10.80$ & $0.344 \pm 0.05$ & 733.4 & 8.50 & -1.15 \\
$\mathbf{W}_{\mathbf{2 2}}$ & $730.0 \pm 6.00$ & $0.384 \pm 0.02$ & 711.6 & 18.30 & -2.57 \\
$\mathbf{W}_{\mathbf{2 3}}$ & $525.0 \pm 10.73$ & $0.307 \pm 0.01$ & 543.3 & -18.30 & 3.36 \\
$\mathbf{W}_{\mathbf{2 4}}$ & $677.0 \pm 9.00$ & $0.402 \pm 0.01$ & 685.5 & -8.50 & 1.24 \\
$\mathbf{W}_{\mathbf{2 5}}$ & $528.0 \pm 5.32$ & $0.227 \pm 0.04$ & 505.1 & 13.05 & -4.52 \\
$\mathbf{W}_{\mathbf{2 6}}$ & $614.0 \pm 7.27$ & $0.345 \pm 0.06$ & 600.9 & -13.05 & -2.17 \\
$\mathbf{W}_{\mathbf{2 7}}$ & $448.9 \pm 7.32$ & $0.242 \pm 0.02$ & 461.9 & -22.85 & 4.82 \\
$\mathbf{W}_{\mathbf{2 8}}$ & $463.7 \pm 3.99$ & $0.374 \pm 0.03$ & 486.5 & -31.36 & 5.30 \\
$\mathbf{W}_{\mathbf{2 9}}$ & $560.0 \pm 5.80$ & $0.392 \pm 0.03$ & 591.3 & -4.55 & 1.14 \\
$\mathbf{W}_{\mathbf{3 0}}$ & $394.1 \pm 10.16$ & $0.292 \pm 0.02$ & 398.5 & 4.55 & -1.06 \\
$\mathbf{W}_{\mathbf{3 1}}$ & $432.5 \pm 12.93$ & $0.311 \pm 0.01$ & 427.9 & -26.00 & -7.75 \\
$\mathbf{W}_{\mathbf{3 2}}$ & $435.7 \pm 12.40$ & $0.391 \pm 0.05$ & 404.3 & 18.00 & 4.96 \\
$\mathbf{W}_{\mathbf{3 3}}$ & $498.0 \pm 11.18$ & $0.226 \pm 0.03$ & 524.0 & 8.00 & -3.43 \\
$\mathbf{W}_{\mathbf{3 4}}$ & $542.0 \pm 10.98$ & $0.361 \pm 0.02$ & 524.0 & 524.0 & -1.52 \\
$\mathbf{W}_{\mathbf{3 5}}$ & $532.0 \pm 13.37$ & $0.292 \pm 0.02$ & & &
\end{tabular}


This indicated that on increasing $\mathrm{X}_{1}, \mathrm{Y}_{1}$ increased. In the present study, high amount of beads imparted the energy to the system was extensively to break down the particles completely. Therefore, activated regions were generated which might be responsible for agglomeration and hence particle size increase on storage. Therefore, less amount of beads generated the energy imparted to the system was enough to cause the complete particle size reduction. Simultaneously, on other side increasing the amount of PVPK-30 or Tween-80 might decrease the particle size. The small particles, which spontaneously aggregated to decrease the surface energy, were stabilized by a layer of surfactant or/and protective polymer. Less amount of stabilizer induced the agglomeration or aggregation and too much stabilizer promoted the Oswald's ripening (Patravale et al. 2004; Patel et al. 2010). Particle size was decreased because the freshly created particulate surfaces were immediately coated by a layer of Tween $80-P V P K-$ 30 , which prevented the broken particles from agglomerating. Concerning $\mathrm{Q}_{1}$, the results of multiple linear regression analysis showed that both the coefficients $b_{1}$ and $b_{3}$ had positive sign while $b_{2}$ had a negative sign $\left(R^{2}=0.9639\right)$. It could be concluded from the equation 4 that $X_{1}$ showed the largest positive effect compare to $\mathrm{X}_{2}$ and $\mathrm{X}_{3}$.

$Y_{2}=84.17+1.81 X_{1}-0.08 X_{2}+1.58 X_{3}-1.31 X_{1} X_{2^{-}}$ $0.03 X_{1} X_{3}-1.71 X_{2} X_{3}-0.96 X_{1}^{2}-2.02 X_{2}^{2}+2.97 X_{3}^{2}$ [4] This indicated that on either increasing $X_{1}$ or $X_{3}$, $\mathrm{Y}_{2}$ increased. As discussed earlier for particle size, small particles surfaces were immediately coated by the layer of surfactant or polymer, which prevented to aggregation. During the dissolution experiments, it was noticed that more amount of PVPK-30 retarded the drug release due to formation of viscous block on to particle surface. The improvement of dissolution rate in nanosuspension was possibly caused by the increased surface area, which enhanced strong hydrophilic character of the drug toward Tween80 via improvement in wettability of the hydrophobic drug.

Therefore, increasing the amount of the beads or the amount of Tween- 80 was expected to increase the drug release after $120 \mathrm{~min}$. The relationship between the dependent and independent variables was further elucidated by constructing the contour plots. The effects of $\mathrm{X}_{1}$ and $\mathrm{X}_{2}$ with their interaction on particle size at a fixed level of $\mathrm{X}_{3}$ are shown in Figure 1. The plots were linear up to $550 \mathrm{~nm}$ particle size, but above this value, the plots were nonlinear indicating a nonlinear relationship between $X_{1}$ and $X_{2}$. It was determined from the contour plot that a lower value of particle size $(500 \mathrm{~nm})$ could be obtained with an $\mathrm{X}_{1}$ level range from -1 to 0 , and an $X_{2}$ level range from -1 to 0.5 . It was evident from the contour graph that the intermediate level of $\mathrm{X}_{1}$ and $\mathrm{X}_{2}$ favored the particle size of nano-suspension. When the coefficient values of two key variables, $\mathrm{X}_{1}$ and $\mathrm{X}_{2}$, were compared, the value for the variable $\mathrm{X}_{1}$ $\left(b_{1}=30.10\right)$ was higher, indicating that it contributed the most to predicting the particle size. The negative effects of $X_{2}$ on the nano-suspension decreased the particle size. Figure 2 shows the contour plot drawn at a fix level of $\mathrm{X}_{2}$. The contour plot of the particle size values were curvilinear and indicated that a 500nm particle size could be obtained for a combination of the two independent variables, the $X_{1}$ level in the range of 1 to 0 , and the $X_{3}$ level in the range of 0 to 1 . The possible explanation was that the Tween- 80 strongly localized at the surface of particles reduced the interface free energy and provided mechanical barrier to coalescence, preventing the aggregation of the particles. Similarly, Figure 3 shows the contour plot plotted at fix level of $X_{1}$. The plot corresponding to $500 \mathrm{~nm}$ was nonlinear, but in a descending pattern with an increase in the amount of the PVPK-30 or Tween-80. The particle size close to $500 \mathrm{~nm}$ could be obtained with an $\mathrm{X}_{2}$ level range of -1 to 0 and an $X_{3}$ level range of 0.5 to 1 . All the contour plots for a particle size close to $500 \mathrm{~nm}$ were nonlinear. This signified that there was no direct linear relationship among the selected independent variables. The aim of the optimization of pharmaceutical formulations is generally to find the levels of the variable that affect the chosen responses and determine the levels of the variable from which a robust product with high quality characteristics may be produced. All the measured responses that may affect the quality of the product should be taken into consideration during the optimization procedure (Holm et al. 2006; Gupta et al. 2010). It was evident from the polynomial equation and contour plots (Figures 1-3) that a high and low level of $X_{1}$ could not target particle size close accordance with $500 \mathrm{~nm}$. 


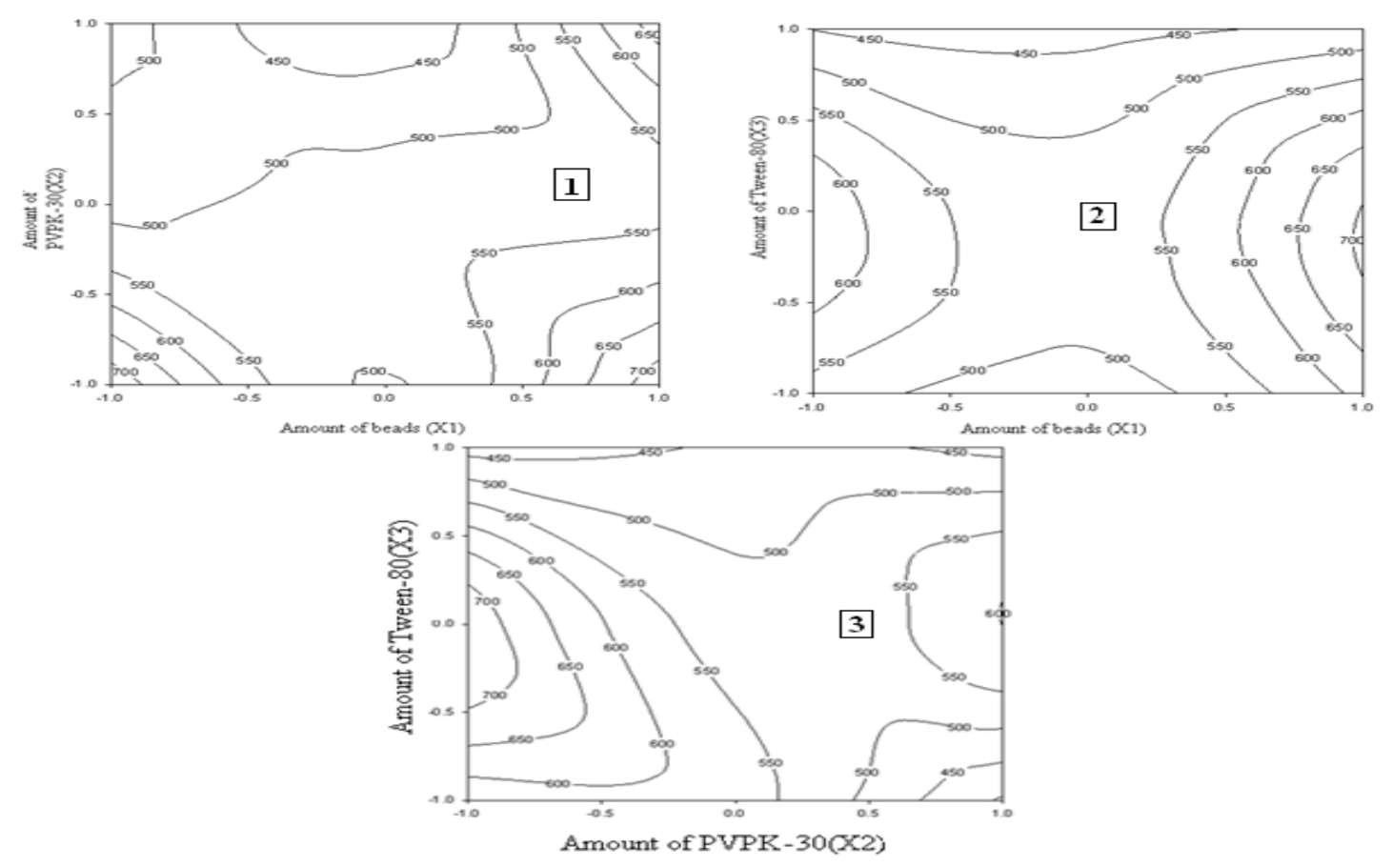

Figures 1-3 - Contour plot showing the effect of amount of beads $\left(\mathrm{X}_{1}\right)$, and amount of PVPK-30 $\left(\mathrm{X}_{2}\right)$ (1), Contour plot showing the effect of amount of beads $\left(\mathrm{X}_{1}\right)$, and amount of Tween-80 $\left(\mathrm{X}_{3}\right)(\mathbf{2})$, Contour plot showing the effect of amount of PVPK-30 $\left(\mathrm{X}_{2}\right)(3)$ on particle $\operatorname{size}(\mathrm{nm})$. The contour lines show particle size.

Hence, the medium level was selected as optimum for $X_{1}$. The optimum formulation targeted the nano-suspension for $500 \mathrm{~nm}$ particle size and more than $80 \%$ drug release within $1 \mathrm{~h}$. Using the desirability function and the contour plots shown in Figures (1-3), 0 level for the amount of the beads $\left(\mathrm{X}_{1}\right)$, a 0 level of the amount of PVPK-30 $\left(\mathrm{X}_{2}\right)$, and a 0.3 level of the amount of Tween-80 $\left(\mathrm{X}_{3}\right)$ were selected as optimal. Using the desirability function, all the defined responses could be combined into one overall response, the overall desirability (Fig. 4). Based on equations (2 and 4), this should be particle size of $500 \mathrm{~nm}$ and drug release after $1 \mathrm{~h}\left(\mathrm{Q}_{1}\right)$ of $84.96 \%$. These calculated values were in close accordance with the experimental results obtained. The experimental results led to particle size of 498.5 $\mathrm{nm}$ and $\mathrm{Q}_{1}$ of $85.24 \%$.

\section{Characterization of optimized famotidine nanosuspension}

The differences of the two famotidine forms were evident also in the morphological studies done by the SEM (Fig. 5). Micronized famotidine powder showed the particle size generally larger (2.5$50 \mu \mathrm{m})$ than the famotidine prepared nanoparticles and had the different morphology. Famotidine micronized suspension crystals showed more irregular elongated shape, while famotidine nanoparticles were more regular and spherical. The media milling of the coarse crystals led to a change of the drug morphology.

Thus, the SEM analyses confirmed a change of drug crystal structure during the wet crushing process. The zeta potential is an important physicochemical characteristic of the nanoparticles. Zeta potential values in therange of $-15 \mathrm{mV}$ to $-30 \mathrm{mV}$ are common for wellstabilized nanoparticles (Pandya et al. 2010; Zidan et al. 2007). Famotidine micronized suspension had -3 mv zeta potential value. This was very less stable suspension due to rapid coagulation or flocculation due to larger particles compared to nano-suspension. Zeta potential of optimized nanosuspension was $-14.8 \mathrm{mV}$ (Fig. 6). Depending on the nature of the interaction of the stabilizers with the drug surface, the properties of adsorbed layer vary the zeta potential. The zeta potential value showed that the PVPK- 30 and Tween- 80 resulted in a complete coverage as they were better able to mask the negative charge on the drug particles. 


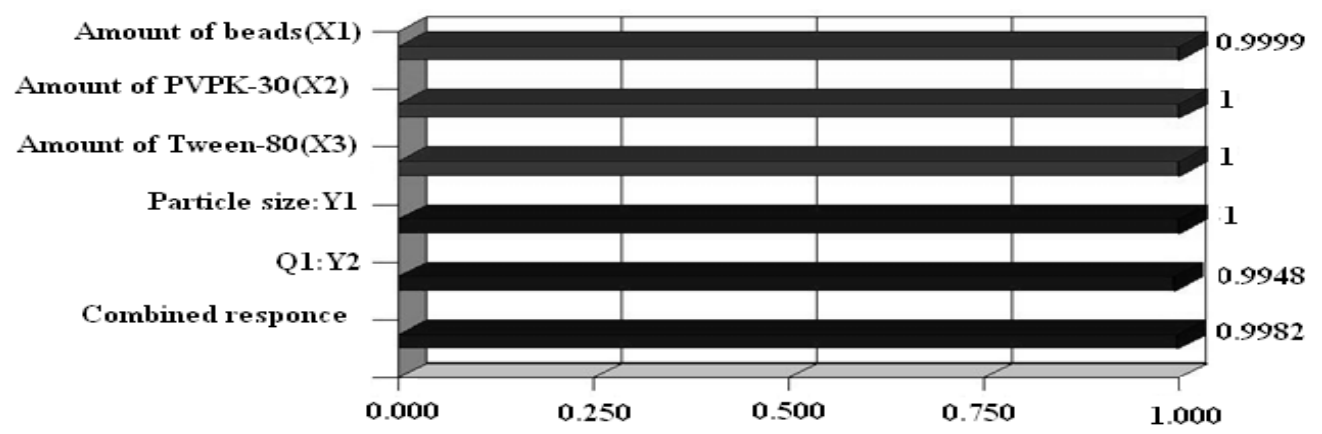

Figure 4 - Bar graph showing individual desirability values of various objective responses and their association overall desirability.
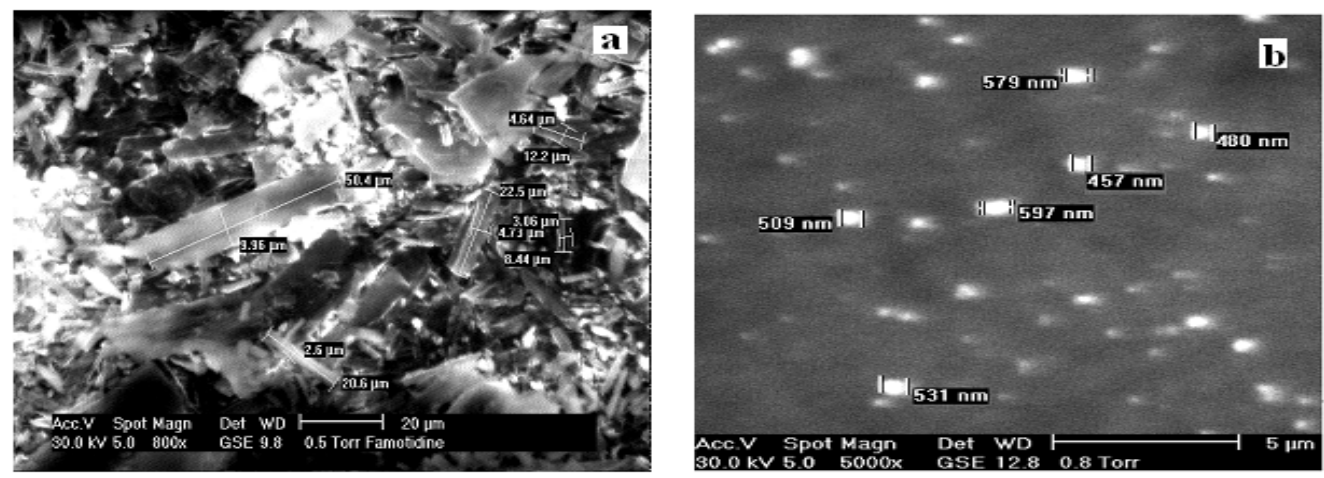

Figure 5 - Scanning electron microscopy images of famotidine micronized suspension (a), famotidine optimized nanosuspension (b).

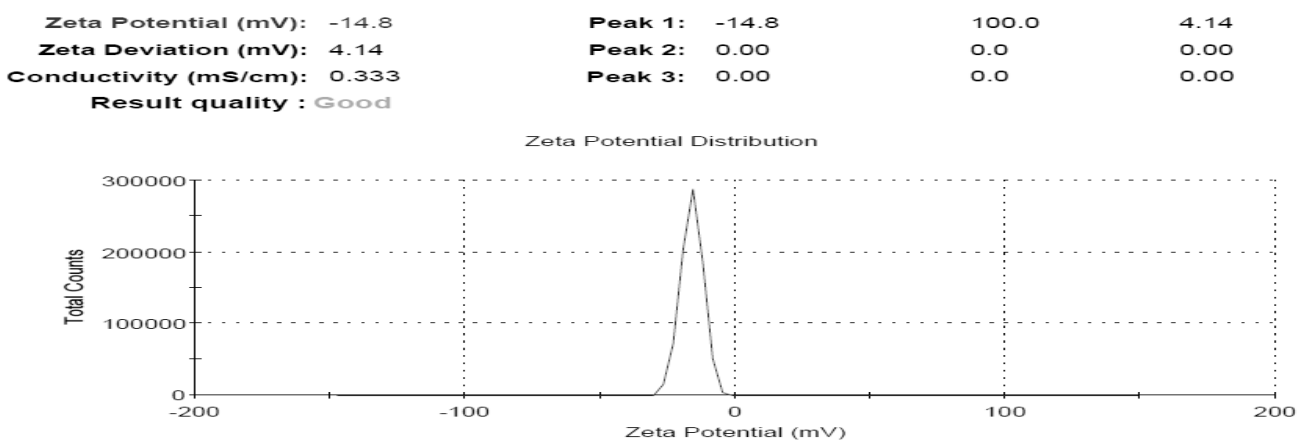

Figure 6 - Zeta potential determination of famotidine loaded optimized nanosuspension.

\section{Rheological behavior}

Viscosity plays a vital role in the dispensing and formulation of nanoparticulate mucoadhesive dispersion. In order to treat the peptic ulcers effectively, the lesion should be in contact with the liquid mucoadhesive formulation for the required time. Therefore, the liquid can readily be applied to the affected region of the mucosa and their high viscosity and mucoadhesion will cause liquid to remain in contact with the lesions for the extended periods. Depending on the need, mucoadhesive nanoparticulate dispersions could be prepared using the individual polymer and binary combinations of HPMCK4M and Na-CMC in variable concentrations. The rheological data shown in Table 7 revealed that all the mucoadhesive nanoparticulate dispersions exhibited shear thickening behavior at low shear 
rates, followed by shear thinning at high shear. Among these formulations, the viscosity was decreased in following order: HPMCK4M/Na$\mathrm{CMC}>\mathrm{Na}-\mathrm{CMC}>\mathrm{HPMCK} 4 \mathrm{M}$. It was also found that HPMCK4M and Na-CMC showed synergistic effect in viscosity enhancing action. The highest viscosity was recorded for the formulation $\mathrm{B}_{3}$ of HPMCK4M/Na-CMC (0.2:0.6\%). A reduction in the concentration of HPMCK4M from formulations $\mathrm{B}_{1}$ to $\mathrm{B}_{3}$ without compromising the rheological properties of the delivery system could be achieved by the addition of viscosity enhancing polymer such as Na-CMC. The HPMCK4M based mucoadhesive dispersions $\left(\mathrm{B}_{4}-\mathrm{B}_{6}\right)$ had fluid like consistency.

Table 7 - Viscosity profile using spindle no. 3 at different rpm for FAM mucoadhesive nanosuspensions.

\begin{tabular}{cccccccccc}
\hline rpm & B1 & B2 & B3 & B4 & B5 & B6 & B7 & B8 & B9 \\
\hline $\mathbf{6}$ & $93.8 \pm 2.95$ & $194.8 \pm 6.11$ & $232 \pm 9.57$ & $83.5 \pm 6.62$ & $90.6 \pm 5.87$ & $95.6 \pm 9.37$ & $89.5 \pm 5.87$ & $93.9 \pm 9.37$ & $110.9 \pm 3.01$ \\
$\mathbf{1 2}$ & $85.7 \pm 1.80$ & $172 \pm 7.68$ & $225 \pm 8.53$ & $72.9 \pm 7.52$ & $76 \pm 6.81$ & $81.8 \pm 6.98$ & $77.2 \pm 6.81$ & $80.1 \pm 6.98$ & $85.7 \pm 4.46$ \\
$\mathbf{3 0}$ & $73.9 \pm 3.16$ & $147 \pm 8.63$ & $203 \pm 8.92$ & $59.6 \pm 8.08$ & $64.8 \pm .82$ & $69 \pm 7.37$ & $65 \pm 6.82$ & $71.7 \pm 7.37$ & $74.9 \pm 5.71$ \\
$\mathbf{6 0}$ & $63.7 \pm 3.58$ & $115.3 \pm 9.96$ & $189 \pm 7.88$ & $48.9 \pm 7.50$ & $54 \pm 8.59$ & $58.3 \pm 3.18$ & $58 \pm 8.59$ & $59.2 \pm 3.18$ & $62.7 \pm 9.13$ \\
\hline
\end{tabular}

Therefore, these could be readily lost from the point of application in peptic ulcer due to the natural movements of the membrane and surrounding structures. This could be attributed that it was hydrated immediately after the contact with the aqueous medium of nanosuspension. Moreover, it was observed that the increase in the polymer (HPMCK4M) level from 0.6 to $0.8 \%$ and to $1 \%$ for $\mathrm{B}_{4}-\mathrm{B}_{6}$ slightly enhanced the viscosity of the nano-suspension. Similarly, Na-CMC content was increased in $\mathrm{B}_{7}-\mathrm{B}_{9}$ formulations, and significant change in the viscosity of nanosuspension was observed. This might be due to change in the amount of the polymer from low to high level. More intimate contact between the particle of Na-CMC results in decreased mobility and the increase in resistance of the gel layer to flow and its erosion. In case of the formulations $\mathrm{B}_{7}-\mathrm{B}_{9}$, the concentration dependency of the viscosity was gradual and as concentration was increased, the systems underwent a sol-gel transition and their viscosity was increased proportionally. When amount of Na-CMC was higher than $0.6 \%$, formulations $\mathrm{B}_{8}$ and $\mathrm{B}_{9}$ were became stiff gels, therefore this could not be able to resuspend the famotidine nanocrytals (Gupta et al. 2010). This finding might suggest that the HPMCK4M hydrated more rapidly than that of HPMCK4M and Na-CMC combination. The formulation $\mathrm{B}_{10}$ did not contain any mucoadhesive polymer and fluid like consistency. It did not generate sufficient viscosity of formulation so it was not essential to identify its results. Additional benefits were not achieved when micronized suspension had higher viscosity due to their bigger particles. It was noticed that they settled down rapidly even though higher viscosity of medium. Hence, the particle size was main governing factor for the physical stabilization of mucoadhesive nano-suspension.

\section{Mucoadhesion measurement}

The combination of ionic polymer such as $\mathrm{Na}$ $\mathrm{CMC}$ and nonionic polymer such as HPMCK4M is known to provide the formulation with desired mucoadhesive properties $\left(B_{3}\right)$ (Singh et al. 2010). The mucoadhesion characteristics were affected by the mixing ratio of the mucoadhesive polymers. The highest detachment force was observed with the formulation $\mathrm{B}_{3}$ followed by $\mathrm{B}_{2}$ and $\mathrm{B}_{1}$ (Fig. 7). This type of interaction results from carboxylic groups of $\mathrm{Na}-\mathrm{CMC}$ and hydrogen binding between the $\mathrm{OH}$ groups of HPMCK4M. Thus, HPMCK4M/Na-CMC dispersions might be adhering rapidly in contact with the hydrated mucous membrane. This may be led to increase the adhesive surface for maximum contact with mucin and flexibility for the interpenetration with mucin. It was noticed that mucoadhesion difference between formulations $B_{1}$ and $B_{2}$ did not reach significant level.

\section{Thermal analysis}

For the bulk material of famotidine, the melting process took place with maximum peak at $167.61^{\circ} \mathrm{C}$. The DSC thermogram of mucoadhesive dispersion showed an endotherm at $121.61^{\circ} \mathrm{C}$, which could be attributed to the melting of the drug (Fig. 8). The peak of famotidine in the formulation showed a slight shift to the lower temperature side. This could be due to a reduction in the particle size and an increase in the surface 
area. The transformation of a sharp to a broad DSC peak due to the melting of famotidine and decrease in the melting point is associated with numerous lattice defects and the formation of amorphous regions in which the drug is located (Pople et al., 2006).

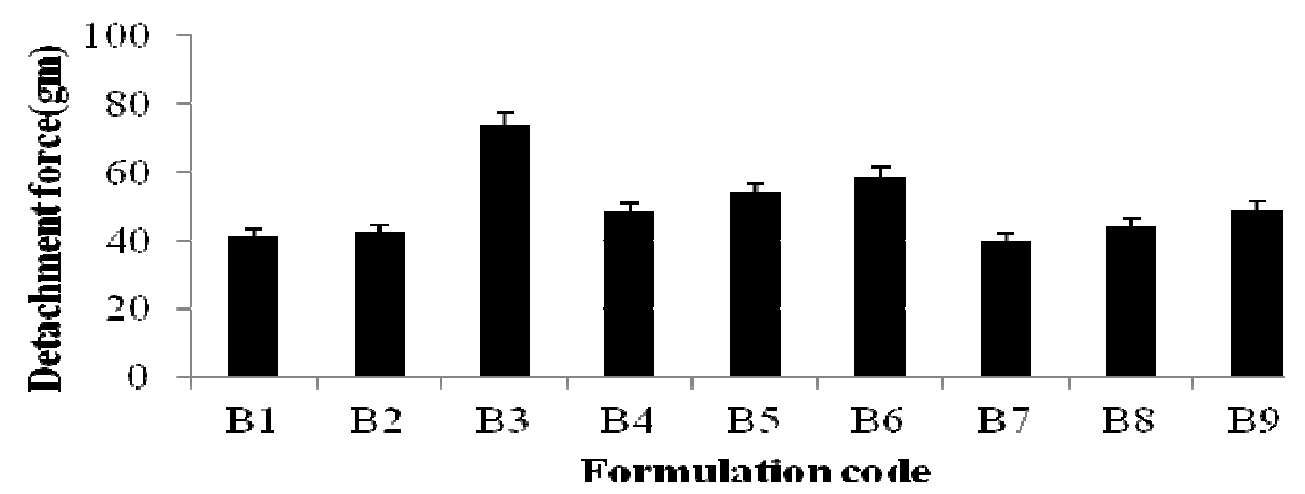

Figure 7 - Bar diagram showing mucoadhesive strength determined as the force of detachment (gm) of mucoadhesive nanoparticulate dispersion (B1 to B9).

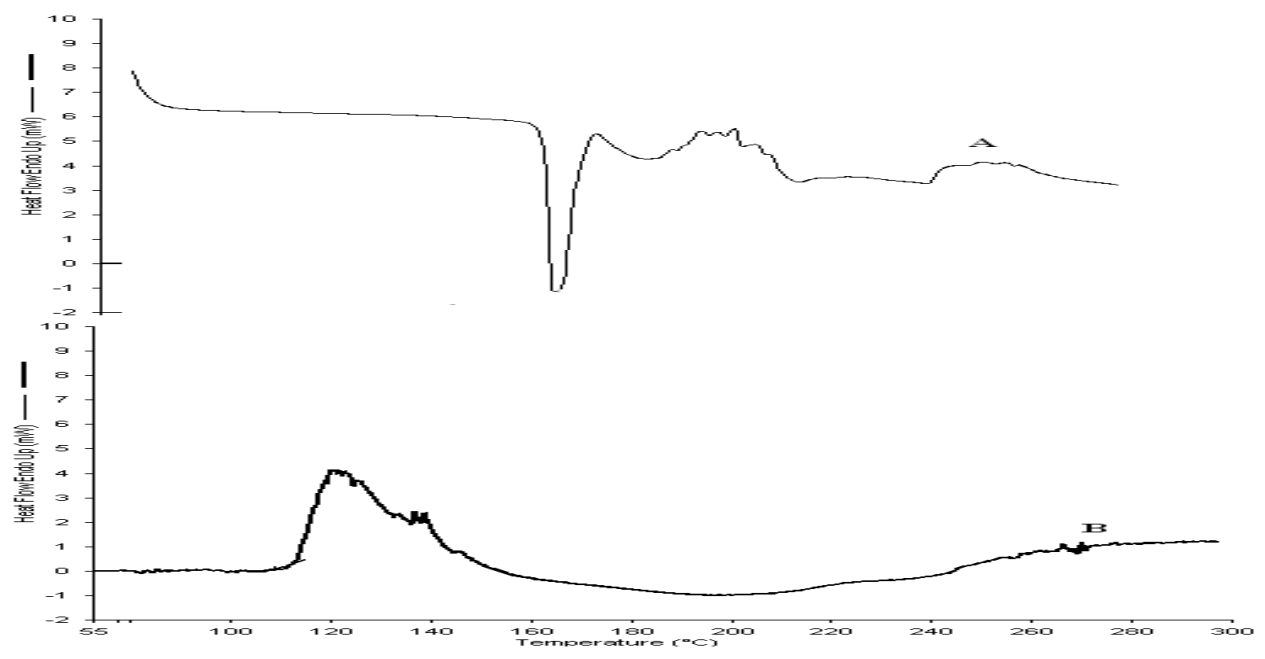

Figure 7 - DSC thermogram of pure famotidine (A), mucoadhesive nanoparticulate dispersion of famotidine (B).

\section{In vitro drug release study}

The cumulative percent of the drug released versus time profiles from the mucoadhesive nanoparticulate dispersion containing HPMCK4M $1 \%(\mathrm{w} / \mathrm{w}), \mathrm{Na}-\mathrm{CMC} 1 \%(\mathrm{w} / \mathrm{w})$ and binary mixture of HPMCK4M/Na-CMC are shown in Figure 9. The results of drug release studies indicated that $\mathrm{B}_{3}, \mathrm{~B}_{6}$, and $\mathrm{B}_{9}$ released 28.07, 33.85, and $46.71 \%$ of drug at the end of $1 \mathrm{~h}$ and 84.49, 94.05, and $97.70 \%$ of drug at the end of $8 \mathrm{~h}$. Among these formulations, the release rate was decreased in the following order: HPMCK4M/Na$\mathrm{CMC}>\mathrm{HPMCK} 4 \mathrm{M}>\mathrm{Na}-\mathrm{CMC}$. These polymers have been well known to retard the drug release by swelling in aqueous media. Although $\mathrm{Na}-\mathrm{CMC}$ and HPMCK4M had similar drug release profile, the release rate was higher with Na-CMC. This could be due to the difference in wettability between the HPMCK4M and Na-CMC which led to a favors in the entrance of water through the polymer (Reddy et al. 2003). The HPMCK4M and $\mathrm{Na}-\mathrm{CMC}$ released the drug at a faster rate than did the HPMCK4M/Na- CMC. These could be attributed to the HPMCK4M/Na-CMC interaction, which facilitates rapid formation of a viscous gel layer upon hydration and this has been regarded as 
an essential first step in achieving the controlled drug release. The results indicated that the formulation $\mathrm{B}_{3}$ had a better ability to retain the drug than the individual polymer in the mucoadhesive nano-suspension and could be used as sustained release drug delivery system in peptic ulcer. The examination of the correlation coefficient ' $\mathrm{r}$ ' indicated that the drug release followed the diffusion controlled mechanism from the mucoadhesive dispersion $\left(\mathrm{B}_{3}\right)$, as the values of ' $r$ ' for zero order (0.9902) were higher in comparison to first order (0.9717) and Higuchi's square root of time (0.9858). To confirm the diffusion mechanism, the data were fit into Korsmeyer's equation. When plotted according to korsmeyer's equation, the formulation $\mathrm{B}_{3}$ also showed high linearity $\left(R^{2}\right.$ 0.9706), with a comparatively slope (n) value of 0.5718 . This $n$ value, however, appeared to indicate a coupling of diffusion and erosion mechanisms, the so-called anomalous diffusion (Singh et al.2010). Hence, the diffusion coupled with erosion could be the mechanism for the drug release from $\mathrm{B}_{3}$ formulation.

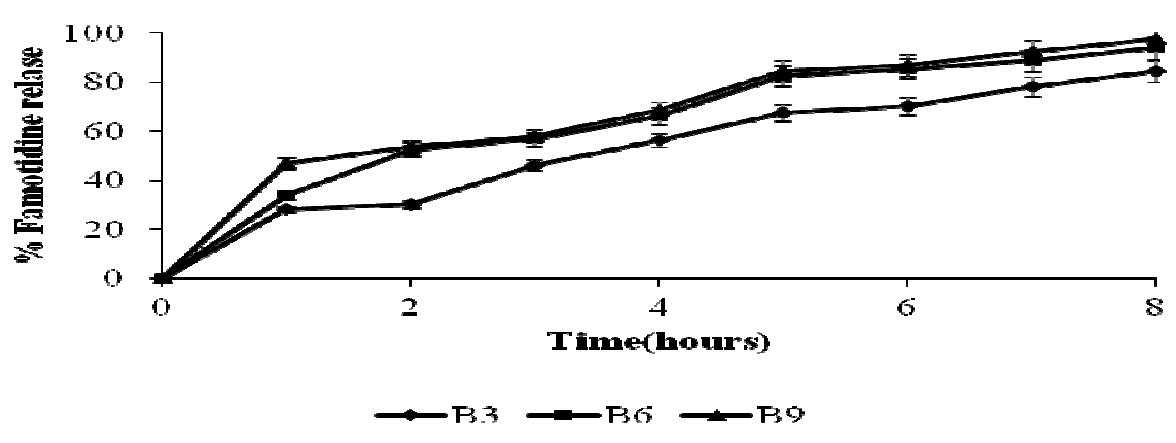

Figure 9 - In vitro release of famotidine from mucoadhesive nanoparticulate dispersion.

\section{Ex vivo retention study}

The mucoadhesive nanoparticulate dispersion $\left(B_{3}\right)$ with highest in vitro mucoadhesivity was compared with famotidine nanoparticles suspension $\left(\mathrm{W}_{33}\right)$ for ex vivo mucoadhesivity study. It was remarkable that formulation $\mathrm{B}_{3}$ showed $75.8 \pm 3.03 \%$ compared to $57.2 \pm 1.64 \%$ mucoadhesion of famotidine nanosuspension after rinsing with $\mathrm{HCl}$-physiological saline. It was noticed that combination ionic polymer (Na-CMC) with non-ionic polymer (HPMCK4M) potentiate the mucoadhesive strength of famotidine nanoparticles (Singh et al. 2010). It could be attributed that hydrogen bond interaction between $\mathrm{Na}-\mathrm{CMC}$ and HPMCK4M promote the mucoadhesive nanoparticles to retain after 6 hours on mucus components of tissue.

\section{In vivo study}

The disease controlled model in rat after the oral administration of aspirin establishes a stable ulcer for at least two days. This permits the characterization of the in-vivo deposition of the particulate carrier system under the influence of gastritis symptoms. After inducing the gastric ulcer, stomach were opened to get visual evidence of the ulceration and to characterize the differences to healthy tissue (Fig. 10). In the histological analyses, strong damages of the gastric tissue were observed. Additionally, it was observed that the stomach wet weight/body ratio increased compared to the healthy control group, which has been known as an indicator for inflammation. The behavior of the proposed nanoparticulate system was examined with respect to reduction in ulcer index and accumulation in the ulcerated gastric tissue after oral administration. Mucoadhesive nanoparticulate dispersion showed significant decrease in ulcer index $(0.46 \pm 0.011)$ when compared with the control group $(3.61 \pm 0.14)$ and famotidine suspension treated animal $(0.66+0.035)$. Qualitatively, an increased adherence of the mucoadhesive nanoparticles was obtained in ulcerated tissue. A size dependant particle deposition in the gastrointestinal tract of healthy subjects as well as mucoadhesion have been reported in the literature depending on the particle surface properties (Hasani et al. 2009). In the present work, famotidine nanoparticulate surface properties were modified with mucoadhesive polymers. Ulcer index of mucoadhesive nanoparticulate dispersion treated 
animal was dramatically reduced compared to famotidine suspension treated animal. It was noticed that mucoadhesive nanoparticles with smallest diameter led to highest adhesion compared to the coarse particle of famotidine suspension. This could be attributed to the strong mucus production in the gastrointestinal tract, especially in the stomach, which favored the particle adhesion to the mucus. Mucoadhesive nanoparticles can better attach to mucus layer due to their small mass.

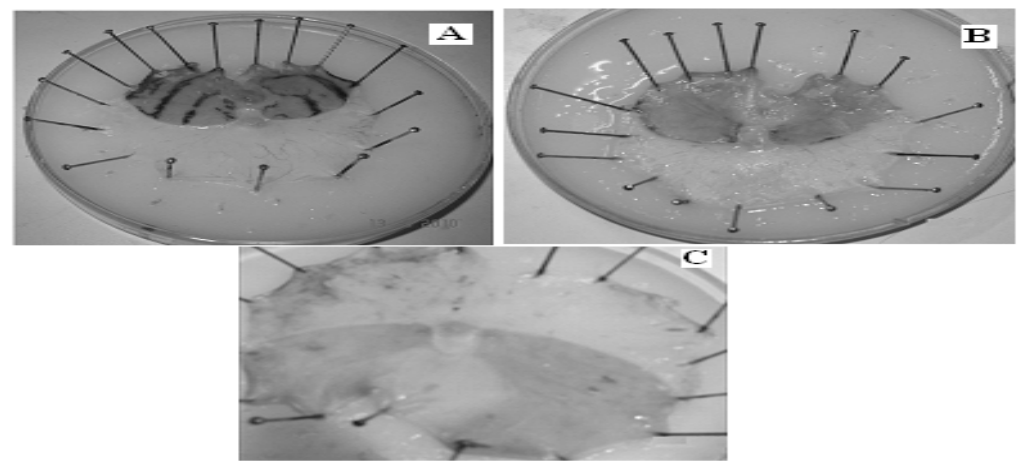

Figure 10 - Induction of ulcer in rat after administration of aspirin treated as control animal (A),famotidine treated rat stomach (B), mucoadhesive nanosuspension treated rat $\operatorname{stomach}(\mathrm{C})$.

\section{Stability study}

Stability studies of the prepared nanosuspensions were carried out by storing the formulation $\mathrm{B}_{3}$ and famotidine nanosuspension at $25 \pm 2{ }^{\circ} \mathrm{C}, 65 \pm 5 \% \mathrm{RH}$ in humidity control oven for three months. The change in the particle size of formulation famotidine nanosuspension and famotidine mucoadhesive nanosuspension were investigated as the function of time to determine whether mucoadhesive polymers had better stabilizing efficiency. Famotidine nanosuspension particle size increased to $573.9 \pm 5.10,638.8 \pm 8.73$ and $734.9 \pm 6.30$ respectively for each month of the analysis from first to third month.

The crystal growth could be due to Ostwald's ripening and was avoided by the addition of mucoadhesive polymers. Upon storage, famotidine mucoadhesive nano-suspension had particle size $578.7 \pm 7.51, \quad 532.7 \pm 4.32$ and $528.9 \pm 3.41$ consecutively for three month analysis. The lower values at third month might be explainable by mucoadhesive polymer having sterically stabilizing effect after adsorption onto particle surface. Since adequate and uniform dosage is a prerequisite for any pharmaceutical suspension, the necessity of controlling the particle sedimentation is obvious. Famotidine nanosuspension showed $0.65 \pm 0.3$ sedimentation volume (SV) ratio compared to $0.85 \pm 0.6$ for the famotidine mucoadhesive after three months.
Therefore, the famotidine mucoadhesive nanosuspension sediment was easily redispersed with soft agitation. On the other hand, famotidine nanosuspension was denser and it was more difficult to resuspend the sediment with vigorous shaking. This could be attributed to the particle size of famotidine nano-suspension, which increased due to Oswald's ripening. The settled particle arranged them into a hard packed cake, which resulted in the failure to obtain good degree of dispersion after three months. In vitro release study of selected mucoadhesive nanosuspension $\left(\mathrm{B}_{3}\right)$ was carried out at $25 \pm 2^{\circ} \mathrm{C} / 65 \% \pm 5 \% \mathrm{RH}$ after three months. Famotidine mucoadhesive nanosuspension was released $90.57 \pm 2.75 \%$ after $8 \mathrm{~h}$. On comparing this data with the previous release data of $\mathrm{B}_{3}$ (Fig.9), it was observed that there was an overall increase in the drug release. These results could be attributed to the erosion of nanoparticles to some extent during the storage (Mishra et al. 2010).

\section{CONCLUSION}

The quality of famotidine loaded nanosuspension was presented using Box-Behnken design and desirability function. In vitro mucoadhesion and in vitro drug release studies clearly indicated that the prepared formulation possessed sufficient 
adhesion and prolong drug release characteristic up to $8 \mathrm{~h}$. Ex- vivo retention of mucoadhesive nanosuspension was higher compared to nanosuspension. Thus, apparently the surface modification of drug nanoparticles improved the retention of drug particles in upper intestinal tract. Moreover, the formulation was therapeutically effective against aspirin induced peptic ulcer. Mucoadhesive nanosuspension containing famotidine nanocrystals could produce added value by allowing a reduction in ulcer index compared to famotidine suspension.

\section{REFERENCES}

Arangoa, MA, Campanero MA, Renedo MJ, Ponchel G, Irache JM. Gliadin nanoparticles as carriers for the oral administration of lipophilic drugs relationships between bioadhesion and pharmacokinetics. Pharm Res. 2001; 18: 1521-1527.

Derle DV, Gujar KN, Sagar, B. Adverse effect associated with the use of non steroidal, antiinflammatory drug: An overview. Indian. J. Pharm. Sci. 2006;68: 409-414.

Gupta S, Samanta MK, Raichur AM. Dual-drug delivery system based on in situ gel-forming nanosuspension of forskolin to enhance antiglaucoma efficacy. AAPS PharmSciTech, 2010;11:322-335.

Hasani S, Pellequer Y, Lamprecht. A Selective adhesion of nanoparticles to inflamed tissue in gastric ulcers. Pharm Res. 2009;5:1149-1154.

Hassan MA, Suleiman MS, Najib NM. Improvement of the in vitro dissolution characteristics of famotidine by inclusion in $\beta$-cyclodextrin. Int. J. Pharm 1990; 58:19-24.

Hawkey, CJ. Nonsteroidal anti-inflammatory drugs and peptic ulcer. British Medicinal Journals, 1990; 300: 278-84.

Holm R, Jensen IH. Sonnergaard J. Optimization of self-microemulsifying drug delivery systems (SMEDDS) using a d-optimal design and the desirability function. Drug Dev. Ind. Pharm 2006:32: 1025-1032.

Ibrahim AA, Rashed S, Rashed Al. Nonsteroidal, antiinflammatory drug-induced gastrointestinal injuries and related adverse reactions: epidemiology, pathogenesis and management. Saudi J Gastroenterol. 2007:13:107-113.

Lai F, Sinicoa C, Ennas G, Marongiua F, Marongiub G, Faddaa AM. Diclofenac nanosuspensions: Influence of preparation procedure and crystal form on drug dissolution behavior. Int. J. Pharm 2009; 373: 124132.

Mishra B, Arya N, Tiwari S. Investigation of formulation variables affecting the properties of lamotrigine nanosuspension using fractional factorial design. DARU. 2010; 18: 1-8.

Patel DJ, Patel, JK. Mucoadhesive effect of polyethyleneoxide on famotidine nanosuspension prepared by solvent evaporation method. Int J Pharm Pharm Sci. 2010; 2:122-127.

Pandya VM, Patel JK, Patel DJ. Improvement in the dissolution of poorly water soluble drug using media milling technique. Thai J Pharma sci. 2010; 34:155164.

Patravale VB, Date AA, Kulkarni RM.. Nanosuspensions: a promising drug delivery strategy. J Pharm and Pharmac 2004; 56:7, 827-840.

Pople PV, Singh, KK. Development and evaluation of topical formulation containing solid lipid nanoparticles of vitamin A. AAPS PharmSciTech 2006; 7:E1-E2.

Rajinikanth PS, Karunagaran LN, Balasubramaniam J, Mishra B. Formulation and evaluation of clarithromycin microspheres for eradication of helicobacter pylori. Chem. Pharm Bull. 2006; 56:1658-1664.

Reddy KR, Mutalik S, Reddy S. Once-daily sustainedrelease matrix tablets of nicorandil: formulation and in vitro evaluation. AAPS PharmSciTech, 2003; 4:19.

Shaikh J, Ankola DD, Beniwal V, Singh, D, Ravi Kumar MN. Nanoparticle encapsulation improves oral bioavailability of curcumin by at least 9-fold when compared to curcumin administered with piperine as absorption enhancer. Eur J Pharm Sci 2009; 37: 223-230.

Singh B, Rani A, Babita, Ahuja N, Kapil R. Formulation optimization of hydrodynamically balanced oral controlled release bioadhesive tablets of tramadol hydrochloride. Sci Pharm 2010; 78: 303323.

Solanki AB, Parikh JR, Parikh RH. Formulation and optimization of piroxicam proniosomes by 3-Factor, 3-Level Box-Behnken design. AAPS PharmSciTech. 2007; 8: E1-E7.

Verma S, Lan Y, Gokhaleb R, Burgessa DJ. Quality by design approach to understand the process of nanosuspension preparation. Int J. Pharm.2009; 377: 185-198.

Zidan AS, Sammourb OA, Hammad MA, Megrab NA, Habib M.J, Khana MA. Quality by design: Understanding the formulation variables of a cyclosporine a self nano emulsified drug delivery systems by Box-Behnken design and desirability function. Int J. Pharm 2007; 332: 55-63.

Received: May 10, 2011; Revised: July 10, 2012; Accepted: December 17, 2012. 